\title{
Ingestão Excessiva de Fitoestrógenos e Telarca Precoce: Relato de Caso com Possível Correlação
}

\section{apresentação de caso}

\author{
ÉRIKA M. FORTES \\ MARTA I. MALERBA \\ PAULO D. LUCHINI \\ EDUARDO K. SUGAWARA \\ LARISSA SUMODJO \\ LUCIANE M. RIBEIRO NeTO \\ IEDA T.N. VERRESCHI
}

Disciplina de Endocrinologia, Departamento de Medicina, Escola Paulista de Medicina, Universidade Federal de São

Paulo, SP

Recebido em 16/11/05

Revisado em 02/03/06 e 09/10/06

Aceito em 22/11/06

\section{RESUMO}

Telarca precoce, desenvolvimento mamário antes dos 8 anos de idade, apresenta dois picos de incidência, nos dois primeiros anos de vida e após os 6 anos de idade. Uma menina de 4,75 anos apresentou-se com telarca associada à ingestão excessiva de fitoestrógenos. $\mathrm{O}$ desenvolvimento puberal M2P1-2, os níveis hormonais e o US pélvico eram compatíveis com puberdade precoce periférica. Durante o seguimento, observou-se ingestão diária de alimentos baseados em soja (> $40 \mathrm{mg}$ fitoestrógenos/dia). Soja contém fitoestrógenos, principalmente genisteína e daidzeína. Embora menos potentes do que o estradiol, sua concentração pode ser 13.000 a 22.000 vezes maior em crianças alimentadas somente com fórmulas baseadas em soja. Os pais foram aconselhados a reduzir para uma vez na semana o seu fornecimento na dieta. O desenvolvimento puberal cessou em M2-3P1 e a paciente, com 8,66 anos, continua a desenvolver-se com idades óssea e cronológica equivalentes. Questões relacionadas à segurança alimentar, principalmente de alimentos baseados em soja, permanecem sem respostas precisas. Embora sejam conhecidas as etiologias da puberdade precoce não progressiva e da telarca prematura, neste caso esteve fortemente relacionada à ingestão excessiva de soja e de outros alimentos ricos em fitoestrógenos que poderiam ter desencadeado a telarca atuando como desreguladores endócrinos. (Arq Bras Endocrinol Metab 2007;51/3:500-503)

Descritores: Pseudopuberdade precoce; Telarca; Desreguladores endócrinos; Fitoestrógenos; Soja

\section{ABSTRACT}

High Intake of Phytoestrogens and Precocious Thelarche: Case Report With a Possible Correlation.

Precocious thelarche is the breast development before 8 years of age with two peaks of incidence during the first two years of life and after 6 years of age. A 4.75-year-old girl presented with thelarche associated to an excessive intake of phytoestrogens (phye). Tanner development B2P1-2, hormonal levels and pelvic US were compatible with peripheral precocious puberty. During follow-up, a daily intake of soy-based foods was observed (> $40 \mathrm{mg}$ phye/day). Soy is rich in phytoestrogens, mainly genistein and daidzein. Although phye are less strong than estradiol, its concentration could be from 13,000 to 22,000 times stronger in children fed only by soybased formulas. Parents were advised and soy intake was reduced to once a week. Progression of pubertal development ceased at B2-3P1. The patient, now 8.66 years old, keeps growing with similar bone and chronological ages. Some questions related to industrial food security, mainly the soy-based food, remain without precise answer. Although it is well known the entity of non-progressive precocious puberty and premature thelarche, pubertal development in this case was strongly related to excessive daily intake of soy and other phye-rich food that could trigger puberty as endocrine disruptor. (Arq Bras Endocrinol Metab 2007;51/3:500-503)

Keywords: Precocious pseudopuberty; Thelarche; Endocrine disruptor; Phytestrogen; Soy 
A

TELARCA PRECOCE É DEFINIDA como o início do desenvolvimento mamário antes dos 8 anos de idade e diagnosticada geralmente durante os primeiros 2 anos de idade, devido à persistência de secreção de gonadotrofinas hipofisárias na infância, e após os 6 anos de idade, devido à antecipação da produção puberal ovariana de estrogênios e/ou o aumento da sensibilidade dos receptores destes hormônios aos baixos níveis circulantes $(1,2)$. Geralmente é uma condição autolimitada, com taxa de regressão variando de 30 a $60 \%$ após um ano e meio de evolução $(3,4)$.

A exposição a estrogênios exógenos pode explicar alguns casos de telarca precoce, sendo uma condição cada vez mais freqüente, porém pouco diagnosticada. Existem relatos de precocidade sexual, tanto em meninos como em meninas, associada ao uso de algumas substâncias como o dietistilbestrol (DES), cremes ou tônicos capilares contendo 17- $\beta$ estradiol e fitoestrógenos (5), bem como uma epidemia de telarca precoce associada ao consumo de fórmulas lácteas à base de soja (6).

A soja, como também outras plantas utilizadas na alimentação humana como cereais (trigo, arroz), leguminosas (feijão, vagem, ervilha, lentilha), frutas (tâmara, romã, cereja, maçã) e chás (7-9), entre outros, são fontes de fitoestrógenos. Essas substâncias, compostos difenólicos não-esteroidais, são importantes desreguladores endócrinos de origem natural.

Relata-se um caso de telarca precoce, onde o provável fator responsável por esse quadro clínico foi o consumo excessivo de fitoestrógenos.

\section{RELATO DO CASO}

A paciente TAS, com 4 anos e 9 meses de idade, iniciou acompanhamento ambulatorial com queixa de desenvolvimento mamário há dois meses, apresentando-se em estágio M2P1-2 do desenvolvimento puberal. A avaliação laboratorial (valores de referência) e de imagem foi compatível com quadro de puberdade precoce periférica. Estradiol $<20 \mathrm{ng} / \mathrm{dl}(<30 \mathrm{ng} / \mathrm{dl})$; testosterona total $38 \mathrm{ng} / \mathrm{dl}(<40 \mathrm{ng} / \mathrm{dl}) ; \mathrm{LH}$ basal $0,2 \mathrm{UI} / 1(<1,5 \mathrm{UI} / \mathrm{l})$ e após $100 \mu \mathrm{g}$ GnRH 0,3 UI/l $(<9,0 \mathrm{UI} / \mathrm{l})$; FSH basal $0,1 \mathrm{UI} / \mathrm{l}(<4,0 \mathrm{UI} / \mathrm{l})$ e pósGnRH 0,2 UI/l; S-DHEA $38 \mathrm{ng} / \mathrm{dl}(<40 \mathrm{ng} / \mathrm{dl})$; androstenediona $2 \mathrm{l} \mathrm{ng} / \mathrm{dl}(<50 \mathrm{ng} / \mathrm{dl}) ; 17 \alpha$-hidroxiprogesterona $33 \mathrm{ng} / \mathrm{dl}(5-100 \mathrm{ng} / \mathrm{dl})$; prolactina $10,8 \mu \mathrm{g} / \mathrm{dl}(<\mathrm{l} 4 \mu \mathrm{g} / \mathrm{dl}) ;$ TSH $1,2 \mathrm{mUI} / 1(0,3-4,0$ $\mathrm{mUI} / \mathrm{l}) ; \mathrm{T} 4$ livre $1,6 \mathrm{ng} / \mathrm{dl}(0,7-1,4 \mathrm{ng} / \mathrm{dl})$. A ultrassonografia pélvica revelava útero com corpo $\mathrm{e}$ colo uterinos medindo respectivamente 3,66 e 0,60 $\mathrm{cm}$, com eco endometrial presente, relação corpo-colo $>\mathrm{l}$; ovário direito medindo $2,54 \times 1,69 \times 2,60 \mathrm{~cm}$ contendo cisto de $19 \mathrm{~mm}$ sugestivo de folículo dominante com parede regular e eco homogêneo; ovário esquerdo medindo $1,06 \times 0,89 \times 0,97 \mathrm{~cm}$.

Durante o acompanhamento, constatou-se situação dietética singular, pelo consumo diário de grande quantidade de alimentos ricos em soja, como farinha caseira com vários grãos (grão de bico, arroz integral, feijão de soja), proteína de soja e linhaça, suplemento comercial à base de soja $\left(\right.$ Soymilk $\left.^{\circledR}\right)$, leite e suco de soja ( $200 \mathrm{ml}$ de cada), soja tostada e shoyu. A partir desta constatação, orientou-se reduzir a ingestão de soja para uma vez por semana. $\mathrm{Na}$ evolução, houve estabilização do desenvolvimento puberal em M2-3Pl e, quando da última avaliação ambulatorial, com 8 anos e 8 meses, a paciente encontrava-se no mesmo estágio do desenvolvimento puberal e mantinha velocidade de crescimento e idade óssea compatíveis com a idade cronológica.

\section{DISCUSSĀO}

Desregulador endócrino é uma substância ou uma mistura de substâncias exógenas que alteram uma ou várias funções do sistema endócrino e têm, conseqüentemente, efeitos adversos sobre a saúde num organismo intacto, sua descendência e/ou subpopulações (10). Podem ser de origem sintética ou natural.

Dentre os fitoestrógenos, desreguladores endócrinos de origem natural, encontrados na dieta humana, destacam-se as isoflavonas e os lignanos (11). As isoflavonas são encontradas em legumes e especialmente na soja e produtos derivados. Esses alimentos apresentam grande quantidade de genisteína e daidzeína que possuem elevadas atividades estrogênicas com maior afinidade pelo receptor $E_{\beta}(12,13)$, podendo agir como agonistas ou antagonistas estrogênicos. São os compostos mais pesquisados por suas potenciais aplicações terapêuticas, assim como os seus metabólitos, o o-desmetilangolesina e o equol $(8,9,14,15)$. Os lignanos são encontrados em cereais, sementes, legumes e em muitas frutas. Esses incluem o metairesinol, o enterodiol e seus produtos de metabolização, o enterolactona e o secoisolariciresinol $(14,15)$. Essas substâncias atuam no metabolismo dos hormônios sexuais e têm demonstrado também uma possível ação como fator de proteção ao câncer $(7,11)$.

Apesar dos fitoestrógenos serem menos potentes que o estradiol, sua concentração pode ser de 13.000 a 22.000 vezes maior em crianças alimentadas exclusivamente com fórmulas lácteas à base de soja (16). 
Tabela 1. Consumo estimado de fitoestrógenos (mg/dia) na dieta da paciente TAS.

\begin{tabular}{lcc}
\hline ALIMENTO & QUANTIDADE ESTIMADA & $\begin{array}{c}\text { FITOESTRÓGENO } \\
\text { (mg/dia) (17) }\end{array}$ \\
Proteína de soja & $20 \mathrm{~g}$ de proteína de soja & 2,4 \\
Soymilk $^{\circledR}$ & $20 \mathrm{~g}$ de leite de soja & 9,7 \\
Ades $^{\circledR}$ & $5 \mathrm{~g}$ de proteína de soja & 0,6 \\
Soja tostada & $25 \mathrm{~g}$ de soja & 32,1 \\
TOTAL & & 44,8 \\
\hline
\end{tabular}

Conduziu-se um levantamento da dieta habitual da paciente em questão. Das informações obtidas, constatam-se muitos alimentos que poderiam atuar como desreguladores endócrinos, sendo que de alguns há informações exatas do consumo diário. Para efeito de cálculo, somente foram considerados os alimentos cujas medidas foram informadas, portanto tomou-se como base a ingestão de proteína de soja, Soymilk ${ }^{\circledR}$, Ades $^{\circledR}$ e soja tostada para se estimar o consumo diário de fitoestrógenos (17) (tabela 1). Vários fatores podem contribuir incrementando ou diminuindo a concentração desses fitoestrógenos, quer sejam aqueles que possam interferir na concentração destas substâncias nas diferentes plantas, tais como a região de cultivo, a variedade, a safra e as condições de armazenamento, além do processo de industrialização e, como no presente caso, a forma de preparo caseiro (18), ou fatores que pudessem modificar a biodisponibilidade dos fitoestrógenos, como na vigência de dieta rica em carboidratos, em parasitoses e doenças intestinais co-existentes e também durante tratamento com antibióticos.

O valor subestimado de $44,8 \mathrm{mg}$ de fitoestrógenos/dia para a dieta da paciente TAS, se comparado a dados compilados na literatura, é no mínimo equivalente ao consumo das crianças alimentadas exclusivamente com fórmulas à base de soja (aproximadamente $40 \mathrm{mg} / \mathrm{dia}$ ) e ao consumo médio dos japoneses ( 25 a $100 \mathrm{mg} / \mathrm{dia}$ ) e que, por sua vez, é maior que o consumo médio da população inglesa (aproximadamente $1 \mathrm{mg} /$ dia) e dos vegetarianos (aproximadamente $3 \mathrm{mg} /$ dia) (17).

Tem sido sugerido que crianças expostas a altos níveis de genisteína presentes em alimentos à base de soja possam sofrer repercussões no trato reprodutivo (13). Este argumento tem sido baseado em dados obtidos com camundongos imaturos expostos a genisteína, que revelaram um aumento dose-dependente de folículos ovarianos $(13,19)$.

Embora sejam bem conhecidos os quadros de puberdade precoce não-progressiva e de telarca prematura, o desenvolvimento puberal neste caso ocorreu na vigência de cisto ovariano, e o quadro regrediu quando corrigida a ingestão excessiva de soja e outros alimentos ricos em fitoestrógenos capazes de desencadear a puberdade atuando como desreguladores endócrinos.

Há relatos de fitoestrógenos desencadeando epidemia de puberdade precoce (6) e vários estudos em animais, nos quais estas substâncias levaram à infertilidade em ovelhas $(20,21)$, diminuição da distância ano-genital e alteração do comportamento sexual em ratos $(22)$.

A partir dessas informações e evidências, como do presente relato, surgem perguntas para as quais ainda não se têm respostas, como referentes à segurança de produtos alimentícios industrializados, principalmente alimentos para lactentes à base de soja, evidenciando a necessidade de determinar-se que substâncias poderiam agir como desreguladores endócrinos interferindo no desenvolvimento puberal antecipando a puberdade.

\section{REFERÊNCIAS}

1. Lee P. Puberty and its disorders. In: Lifshitz F. Pediatric Endocrinology. $4^{\text {th }}$ ed. New York: Marcel Decker, 2003. pp. 211-35.

2. Pasquino AM, Piccolo F, Scalamandré A, Malvaso M, Ortolani R, Boscherini B. Hypothalamo-pituitary-gonadotropic function in girls with premature thelarche. Arch Dis Child 1980;55:941-4.

3. Aritaki S, Takagi A, Someya H, Jun L. A comparison of patients with premature thelarche and idiopathic true precocious puberty: in the initial stage of illness. Acta Pediatr Jpn 1997;39:21-7.

4. Mills JL, Stolley PD, Davies J, Moshang T. Premature thelarche: natural history and etiologic investigation. Am J Dis Child 1981; $135: 743-5$.

5. Nebesio TD, Pescovitz OH. Historical perspectives endocrine disruptors and the timing of puberty. Endocrinology 2005; $15: 44-8$.

6. Freni-Titulaer LW, Cordero JF, Haddock L, Lebron G, Martinez R, Mills JL. Premature thelarche in Puerto Rico. A search for environmental factors. Am J Dis Child 1986;140:1263-7.

7. Clapauch R, Meirelles RMR, Julião MASG, Loureiro CKC, Giarodoli PB, Pinheiro AS, et al. Fitoestrógenos: posicionamento do Departamento de Endocrinologia Feminina da Sociedade Brasileira de Endocrinologia e Metabologia (SBEM). Arq Bras Endocrinol Metab 2002;46:679-95.

8. Gruber CJ, Tschugguel V, Schneeberger C, Huber JC. Production and actions of estrogens. $N$ Engl J Med 2002;346:340-52. 
9. Adlercreutz M. Phytoestrogens: epidemiology and a possible role in cancer protection. Environ Health Perspect 1995; 103:103-12.

10. European Commission, DG XXIV, Consumer Policy and Consumer Health Protection. Scientific Committee for Toxicity, Ecotoxicity and the Environment. Estee opinion human and wildlife health effects of endocrine disrupting chemicals, with emphasis on wildlife and on ecotoxicology test methods. Available at: <http//www. Europa.eu.int/ comm./ dg24/ health>. Accessed in March 1999.

11. Haddad NG, Fuqua JS. Phytoestrogens: effects on the reproductive system. Endocrinologist 2001;11:498-505.

12. Kuiper GGJM, Lemmen JG, Carlsson B, Corton JC, Safe SH, Van Der Saag G PT, et al. Interaction of estrogenic chemicals and phytoestrogens with estrogen receptor $\beta$. Endocrinology 1998; $139: 4252-63$

13. Jefferson WN, Couse JF, Padila-Banks E, Korach KS, Newbold RR. Neonatal exposure to genistein induces estrogen receptor (ER) $\alpha$ expression and multioocyte follicles in the maturing mouse ovary: evidence for ER $\beta$-mediated and nonestrogenic actions. Biol Reprod 2002;67:1285-96.

14. Segundo informe nacional sobre la exposición humana a compuestos químicos ambientales. Departamento de salud y servicios humanos centros para el control y la prevención de enfermidades (CDC), p. 1-4, 145158, 194-195, enero 2003.

15. Greim AH. The endocrine and reproductive system: adverse effects of hormonally active substances? Pediatrics 2004;113:1070-5.

16. Setchell KD, Zimmer-Nechemias L, Cai J, Heubi JE. Exposure of infants to phyto-estrogens from soy-based infant formula. Lancet 1997;350:23-7.

17. Committee on Toxicity of Chemicals in Food, Consumer Products and the Environment Phytoestrogens and Health 2003, capítulo 4. Available at: <http://www.food.gov.uk/ multimedia/pdts/phyto_report0503>.
18. Zung A, Reifen R, Kerem Z, Zadik Z. Phytoestrogens: the pediatric perspective. J Pediatr Gastroenterol Nutr 2001;33:112-8.

19. Jefferson WN, Padilla-Banks E, Newbold RR. Adverse effects on female development and reproduction in CD-1 mice following neonatal exposure to the phytoestrogen genistein at environmentally relevant doses. Biol Reprod 2005. Available at: <http://www.biolreprod.org/cgi/rapidpdf/biolreprod.105.041277v1>

20. Moule GR, Braden AWH, Lamond DR. The significance of oestrogens in pasture plants in relation to animal production. Anim Breed Abstr 1963;31:139-57.

21. Shutt DA. The effects of plants oestrogens on animal production. Endeavour 1976;35:110-3.

22. Wisniewski AB, Cernetich A, Gearhart JP, Klein SL. Perinatal exposure to genistein alters reproductive development and aggressive behavior in male mice. Physiol Behav 2005;84:327-34.

\section{Endereço para correspondência:}

leda T.N. Verreschi

Lab. de Esteróides

Disciplina de Endocrinologia

Depto. de Medicina da Universidade Federal de

São Paulo - UNIFESP/EPM

Rua Pedro de Toledo 781, 13\% andar

04039-032 São Paulo, SP

E-mail: ieda@endocrino.epm.br 